\title{
VISUALIZING 3D CLIMATE DATA IN URBAN 3D MODELS
}

\author{
J. Gautier*, S. Christophe, M. Brédif
}

LASTIG, Univ Gustave Eiffel, ENSG, IGN, F-94160 Saint-Mande, France - (jacques.gautier, sidonie.christophe, mathieu.bredif)@ign.fr

\author{
Commission IV, WG IV/9
}

KEY WORDS: 3D visualization, co-visualization, urban climate, climate visualization, simulation data, 3D field data.

\begin{abstract}
:
In order to understand and explain urban climate, the visual analysis of urban climate data and their relationships with the urban morphology is at stake. This involves partly to co-visualize 3D field climate data, obtained from simulation, with urban 3D models. We propose two ways to visualize and navigate into simulated climate data in urban 3D models, using series of horizontal 2D planes and 3D point clouds. We then explore different parameters regarding transparency, 3D semiologic rules, filtering and animation functions in order to improve the visual analysis of climate data 3D distribution. To achieve this, we apply our propositions to the co-visualization of air temperature data with a 3D urban city model.
\end{abstract}

\section{CONTEXT : ANALYSIS OF URBAN CLIMATE PHENOMENA.}

Understanding urban climate dynamics is one of the issues regarding global warming. Urban population (more than $50 \%$ of the global population) is particularly more vulnerable to the temperature rise, because of the urban heat island (UHI) phenomena, which causes higher temperatures in cities than in rural areas (Ramyar et al., 2019). UHIs have been a subject of research for many years (Akbari, Kolokotsa, 2016), and continue to be studied (Bouyer et al., 2011, Plumejeaud-Perreau et al., 2015, Masson et al., 2014, Ramyar et al., 2019, Parsaee et al., 2019, Equere et al., 2020) for the development of urban planning and public policies which could mitigate their impacts.

The representation of climate data obtained through simulation models such as Meso-NH (Lac et al., 2018) and TEB (Masson, 2000), into visualization environments allowing an exploratory visual analysis and incorporating other spatial data, presents several advantages for the study of UHIs. Visualizing them in an urban environment allows to spatially contextualize them (Pinson, Masson, 2016), providing a better understanding of the bounds of the urban heat islands, and helping to identify the most impacted areas. The visualization of these simulated data also allows estimating the possible evolution of urban heat islands caused by urban planning modifications or new public policies, by co-visualizing different simulation scenarios. Finally, a co-visualization of the outputs of climate simulation models with information about urban morphology, used (or not) as an input of the simulation models, could provide a visual analysis of breaks or inconsistencies in the simulated data, which could lead to an improvement of the simulation models.

Reaching these different objectives requires to provide visualization methods allowing to represent climate data in their urban spatial context, to represent their spatial evolution in several dimensions, and to co-visualize them with other information (urban design forms, albedo values, land uses property.. etc). Visualizations of geospatial climate data are often proposed through 2D plane representations enabling a multiscale visualization (Pinson, Masson, 2016), some of them providing a dynamic change when displaying climate data according

\footnotetext{
* Corresponding author
}

to the altitude elevation ${ }^{1}$. Analyzing the relationships between urban morphology (urban canyon depth and direction, building heights) and climate data implies to represent the distribution of the data, and their spatial context, according to three spatial dimensions. Such representations raise two main issues:

- the visualization of 3D spatial scalar fields and vector fields, such as temperature or wind data;

- their co-visualization with a 3D urban model.

Proposing visualizations of 3D climate data, and their co-visualization with other spatial data, requires to focus on the issues arising from the graphic representation of 3D objects, such as 3D semiology and 3D data occlusion when co-visualizing various sets of 3D data. We present here our methodology in the exploration of different graphic representation methods to obtain these visualizations. By exploring different visualization methods for the visual analysis of 3D complex simulated data (complex simulation models, multi-scale data) regarding urban climate phenomena, this paper is part of a long-term research approach to bring simulation and visualization more closer.

\section{RELATED WORKS.}

In this section, we present the nature of the climate data we are dealing with, and the advantages of using 3D visualization environments for the visual analysis of such data. Then, we present different works handling with the graphic representation of climate data. First, we focus on the question of climate data semiology, and particularly on the use of color to improve the visualization of climate data, as well in 2D representations as in $3 \mathrm{D}$ representations. Then we present different propositions of climate data visualization, which could be used as inspiration for the co-visualization of 3D climate data with 3D city models, and we discuss the advantages and limits of these propositions.

\footnotetext{
1 www.windy.com
} 
Climate simulation issues. The causes of urban heat islands are diverse (Oke, 2002, Akbari et al., 2001, Santamouris, 2001, Bouyer et al., 2011): mineralization of cities, lower albedo causing more solar energy absorption, building density, reduced air velocity caused by higher urban surface roughness (Masson et al., 2014, Plumejeaud-Perreau et al., 2015). A better understanding of the urban heat islands needs a more accurate knowledge of urban morphology (Stewart, Oke, 2009, PlumejeaudPerreau et al., 2015). As an example, (Stewart, Oke, 2012, Rodler, Leduc, 2019) proposed to use a finer description of the urban space through the Local Climate Zone (LCZ) classification, defining regions of uniform surface cover, structure, material, and human activity. Several works extended the LCZ classification, by taking into account land use or socio-economic criteria (Plumejeaud-Perreau et al., 2015). Simulation models use this description of the urban space, in order to model temperature, wind, or humidity values. These simulations allow to calculate the possible bounds of urban heat islands, but also provide a way to predict the effect of a possible urban planning policy on the evolution of the urban heat islands, providing help for the mitigation of their effects (Bouyer et al., 2011). Amongst others, two models simulate climate data for the analysis of climate phenomena related to urban environments (Pinson, Masson, 2016, Oke, 1976, Renard, Alonso, 2017):

- the Meso-NH model (Mesoscale Non-Hydrostatic atmospheric model), developed by the Laboratoire d'Aérorologie (UMR 5560 UPS / CNRS) and the CNRM (UMR 3589 CNRS / Météo-France), simulates climate data for the atmosphere until altitudes of 15000 meters, and allows an analysis of the urban boundary layer ICU (Oke, 1976, Renard, Alonso, 2017), located above the roofs of the buildings;

- the TEB model (Town Energy Balance), developed by the CNRM (UMR 3589 CNRS / Météo- France), allows to extend Meso-NH simulations in the urban environment, below the buildings' roofs, allowing an analysis of the surface ICU and the urban canopy layer ICU (Oke, 1976, Renard, Alonso, 2017) located between the ground and the roof of the buildings.

Using 3D visualization environments, while providing suitable graphic representations of the evolution of simulated climate data, according to the three dimensions of space, appears to be a possible support for the visual analysis of the different ICU (surface, urban canopy layer, urban boundary layer). The covisualization of climate 3D datasets with $3 \mathrm{D}$ urban models, and morphological indicators such as LCZ could support a visual analysis of the relationships between climate data and urban morphology. We present here a state of the art of different visualization techniques that could be used to perform a covisualization of simulated climate data with a 3D urban model. We focus on the graphic 3D representation of the climate data with a 3D city model, but not on the graphic representation of the 3D model itself.

Visualizing simulated climate data raises several questions already, such as the visualization of the uncertainties related to these simulated data, which can be caused by the imprecision of the input data, and the uncertainty directly linked to the simulation models. Several works have been done on the visualization of uncertainties (Arnaud, Davoine, 2009, Rautenhaus et al., 2017, Johannsen et al., 2018), and can be used through visualization methods for climate data. However, this aspect of data visualization is not addressed in this paper.
The use of color in climate data visualization. Different works propose to display climate data into urban 3D models by using the color of $3 \mathrm{D}$ objects to display data values. To achieve this, 3D visualization can use color to represent scalar climate data such as temperature, as it is already used in 2D representations. In these approaches, climate data such as temperature are mainly represented through rainbow color scales using through RGB (Red, Green, Blue) color palette, even if the efficiency of these scales has already been criticized for cartography and visual analysis (Brewer, 1997, Borland, Taylor, 2007, Stauffer et al., 2015, Dasgupta et al., 2018). One of the most criticized things about the representation of scalar data distribution with rainbow color scales is the fact that the colors may show luminance gradients, which do not correspond to the represented data value gradients. Regarding this problem, some propositions have been made to propose alternative color scales, respecting a continuous evolution of luminance, notably through the use of alternative color palettes such HCL (Hue, Chroma, Luminance). User tests showed that continuous evolution of luminance is more efficient to interpret differences of magnitudes in climate data than a hue evolution, but that a hue evolution is more efficient to interpret differences in spatial distribution (Dasgupta et al., 2018). Other user tests show that color scales using progressive evolution in both hue and luminance, by showing an evolution between two opposite hues with low luminance through a neutral white/grey color with high luminance, allow a better interpretation than rainbow color scales for the analysis of magnitudes and spatial distribution in climate data (Stauffer et al., 2015). Other works remind that the choice of these opposite colors has to respect certain conventions to avoid misinterpretation (Schneider, Nocke, 2018), such as the use of blue and red to respectively represent cold and hot temperature values. However, despite user tests showing the better efficiency of such scales on the analysis of climate data, and good users feedbacks on the efficiency of those scales, a lot of climate scientists still consider they are confident with rainbow color scales (Dasgupta et al., 2018). An interactive (3D) visualization of climate data should then give the possibility to dynamically change between rainbow color scales, and other color scales based on a continuous evolution of luminance.

Integration of 3D climate data in 3D visualization environments. As previously said, climate data can be displayed into urban 3D models by using the color of 3D objects, such as the buildings and the ground of the 3D models. Scalar values can then be represented with color. Fig.1 shows different examples of such representation:

- Representations in Fig.1.A and Fig.1.B show the visualization of the horizontal evolution of 2D scalar data, and the co-visualization of this data with the building density, shapes and heights:

- Air quality data (2D data) are represented by the ground color (Kurppa et al., 2020) (Fig.1.A);

- Air temperature data at 2 meters above ground (2D data) are represented by the color on both the ground and the 3D buildings (Bouyer, 2009) (Fig.1.B).

- Fig.1.C shows the visualization of a 3D scalar data according to three directions. Differences between simulated temperatures of the buildings surface and measured outdoor temperature (3D data) are represented by the color on both the ground and the 3D buildings (Pinson, Masson, 2016) (Fig.1.C). Buildings color could also be used, like in 
Fig.1.C, to represent 3D climate data like air temperature, the vertical evolution of the data being represented along the building facades.

Several 2D visualizations of climate vector data such as wind use animated glyphs (Ware et al., 2016, Lobo, Hurter, 2019), the movement of animated particles representing the flow direction, and the speed, the size or the color of the particles representing the flow intensity. These animated glyphs can be used, like color, to represent climate data on the color of 3D objects.

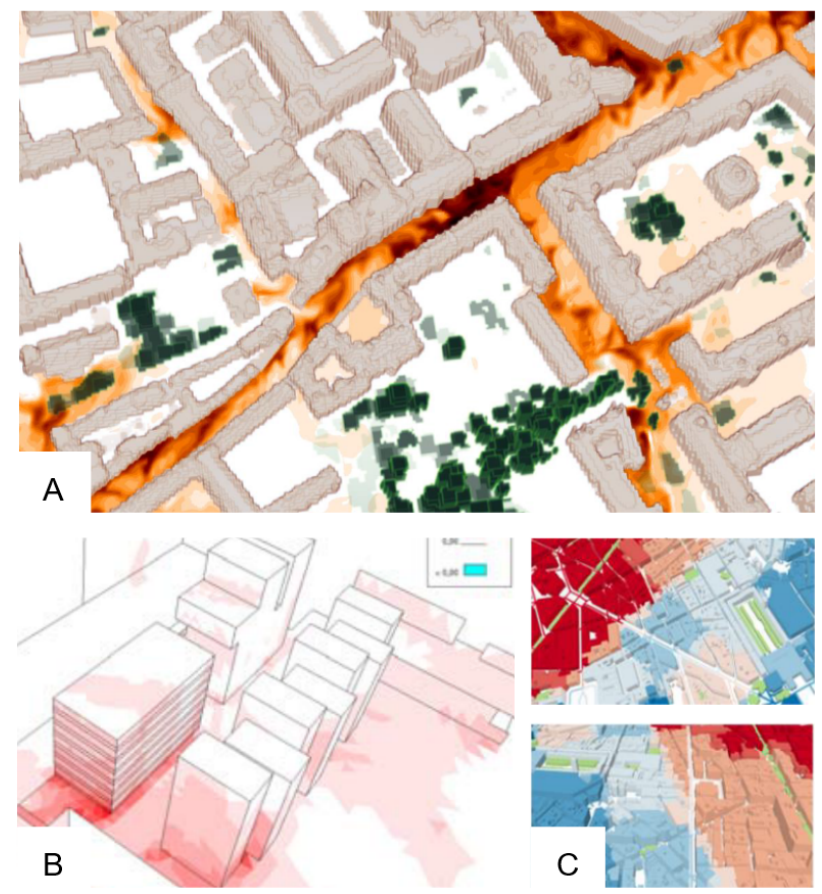

Figure 1. Using ground and building color to represent 2D and 3D scalar data; (A) Air quality data represented by the color of the ground (Kurppa et al., 2020); (B) Representation of the gap between simulated building surface temperature and meteorologic air temperature data (Bouyer, 2009); (C) Using colored buildings to represent simulated air temperature data close to the ground surface (Pinson, Masson, 2016).

Other works propose to represent 3D climate data through one or several 2D planes. The Fig.2.A shows an example of the representation of the wind speed according to one direction (scalar $3 \mathrm{D}$ data). The wind speed distribution is represented on a $2 \mathrm{D}$ vertical plane. The wind speed value is represented on the plane by color. In order to see how the wind is distributed at any place in the city, the $2 \mathrm{D}$ vertical plane is animated and moves along the city along an orthogonal axis.

Propositions, not originally developed for this purpose, could be extended for similar representations of 3D climate data. Some works propose a version of the space-time cube (Aigner et al., 2011), taking the shape of a 3D stack of 2D planes (Fig 2.B), to represent several raster data related to different time steps. The work of (Spur, Tourre, 2018) proposes a similar visualization of several thematic geospatial 2D data by representing each of them on a 2D horizontal plane representation, and displaying each plane one on top of the other in a 3D environment, forming a 3D stack of 2D planes (Fig 2.C). These propositions could be used to extend the representation of $3 \mathrm{D}$ data as series of
2D data, which is used in certain $2 \mathrm{D}$ representations ${ }^{2}$ : a series of $2 \mathrm{D}$ climate datasets corresponding to several altitude levels could be represented on a series of 2D planes, and integrated in a 3D urban model. Data distribution can be represented by the color of the 2D planes.
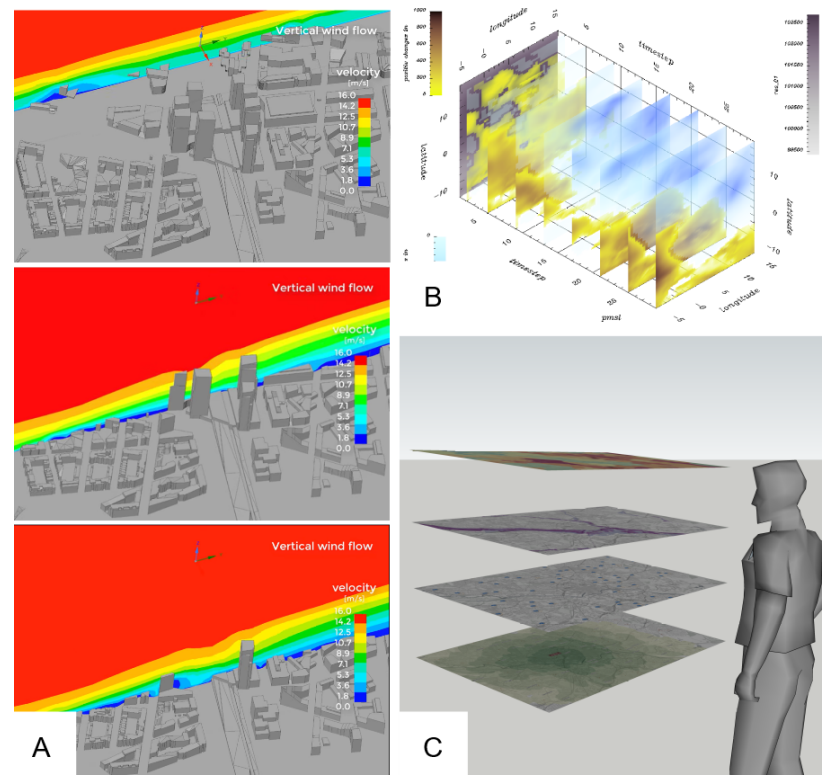

Figure 2. Using one or several 2D planes to represent 3D scalar data; (A) Wind speed data rendered on a spatially animated 2D vertical section (Image from the Kalasatama Digital Twins Project, City of Helsinki ${ }^{3}$ ); (B) Using 3D space-time cube to represent 2D raster data at different timesteps (Aigner et al., 2011); (C) Using 3D stack of 2D planes to represent several thematic data. (Spur, Tourre, 2018).

Another proposition is to graphically represent $3 \mathrm{D}$ data as $3 \mathrm{D}$ fields, with 3D textures using transparency or filtering to highlight the higher values of the 3D field, or with 3D fields of separated particles in order to let spatial objects be visible through the climate data thanks to the empty space between particles:

- In the Fig.3.A, the wind speed according to one direction (scalar data) is represented by a continuous gradient or color and transparency, forming a kind of fog in the $3 \mathrm{D}$ representation. High values are represented by opaque dark colors and the low values by light and transparent colors.

- In the Fig.3.B, wind flows (vector data) are represented by 3D fields of animated glyphs, direction being represented by the movement of the particles, speed being represented by the color, the size, and the speed of the particles $(\mathrm{Li}$, Wang, 2017).

Advantages and limits of these representations. Using the color of 3D buildings to display climate data could be a way to co-visualize these data with the urban shapes, but it presents some limits:

- It could lead the user to interpret that the represented data is related to the building itself (as the temperature of building surface), which is not the case in the representation of the air temperature.

\footnotetext{
2 www.windy.com

3 www.hel.fi/helsinki/en/administration/information/general/3d/3d
} 


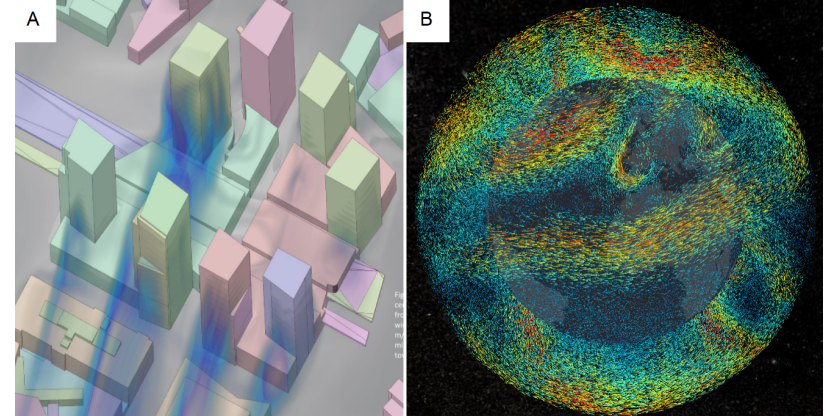

Figure 3. Representing 3D data as 3D fields; (A) using 3D continuous texture to represent 3D data. (Image from the Kalasatama Digital Twins Project, City of Helsinki); (B) using $3 \mathrm{D}$ fields of animated glyphs to represent $3 \mathrm{D}$ wind data $(\mathrm{Li}$, Wang, 2017).

- It would not allow to represent vertical data distributions in areas where there is no buildings such as urban canyons or parks, as well as above the buildings. Using building color to represent climate data could be used to visually analyze the surface and urban canopy layer UHIs (located below the buildings roofs), but would not provide a visualization of the urban boundary layer UHIs (located above the buildings roofs), and the possible relationships between these two levels (Oke, 1976, Renard, Alonso, 2017).

Representing climate data through auxiliary objects, or 3D graphic fields, offers two alternative ways to co-visualize climate data, according to the three dimensions of space, with a 3D urban model.

Representing climate data through 2D plane(s) could be a way to distinguish the displayed values from the building, and to represent data vertical distribution in areas without buildings. In the Fig.2.A, the displayed wind data are related to only one 2D section of the space, but the movement of the displayed vertical section allows to represent data distribution along the city. The use of animation may impact the readability of the visualization (Kaddouri et al., 2014). If the animation allows to perceive a gradient in the data distribution, it is less efficient for comparing data distributions corresponding to two states of the animation (two places in the city in the case of Fig.2.A). Using several 2D planes could be a way to represent climate data without using animation functions. Extending the work of (Spur, Tourre, 2018) could be a way to use several 2D horizontal cross sections to represent a $2 \mathrm{D}$ distribution of climate data at several altitude levels. Allowing the co-visualization of the urban model and the data supported by the different auxiliary objects, and avoiding the graphical occlusion of data, could remain an issue.

Representing climate data through 3D graphic fields could be another way to represent the data distribution according to the three direction of space, and could be a better way to visualize the volume corresponding to the climate data. The main issue is there to allow the user to see other spatial datasets through the 3D graphic field. Using a continuous gradient, or color and transparency like in the Fig.3.A, avoids to hide the 3D urban model behind the climate data, and allows to show the location of higher values in a 3D space. However, the representation focuses on the highest values and makes it difficult to see the spatial evolution of data for the lower values. By representing the flows by particles separated by empty space, the representation used in Fig.3.B shows wind data at different altitude levels, each being represented one behind the other. Incorporated in a 3D urban model, such representation would allow to display different levels of climate data one behind the other, and let the buildings visible through the blank space. By using the color of the particles, this representation could show the distribution of the data values in a 3D space for its entire value scale. However, the density and the size of the particles will intervene on the visibility of different data, through the empty spaces of the fields of particles.

\section{METHODOLOGY.}

We propose our own co-visualizations of 3D climate data with a 3D urban model, in order to provide a visual analysis of climate data distribution in the three dimensions of space, and its relationships with urban morphology. We focus here on the representation of 3D scalar data such as air temperature data, and we use color to represent scalar data value, by following the guidelines presented in the previous section. To represent the 3D urban model, we use an abstract representation, by only considering the footprint and the height of each building.

In order to visualize the distribution of scalar climate data, along the three dimensions of space but also on their entire scale of values, we propose to explore three ways of representation and their integration in a 3D urban model: a 2D planes series representation, a 3D point cloud representation, a value-related 3D point cloud representation.

We propose to represent data values through different HCL color scales, respecting the guidelines proposed by (Stauffer et al., 2015) concerning the use of hue and luminance to represent quantitative values. We let the possibility to the user to dynamically change between the proposed color scales. We also let the possibility to choose a rainbow color scale to represent the data values, regarding that a lot of climate scientists consider they are confident with rainbow color scales (Dasgupta et al., 2018).

We propose a geovisualization of climate data at different spatial scales of the urban environment, such as city, and street scales. We first experiment our visualizations at a street scale, where the structure of the streets and the buildings height are more visible, before expand it at a larger scale.

Design of possible integrations of 3D climate data in a 3D urban model. We created three types of co-visualizations of 3D scalar climate data with a 3D urban models, involving different parameters regarding graphic representation and rendering, which could be presented to users such as meteorologists and climate scientifics to validate the relevance of these design propositions for climate data analysis. For each co-visualization, we let the possibility to the user to modify the design parameters (color scale, transparency, etc.), in order to let him define the most suitable ones for each use case.

The 2D planes series representation consists in representing 3D scalar data through a series of 2D slices corresponding each to one height level, and to represent each of these 2D slices with a 2D horizontal plane, forming a series of horizontal 2D planes placed one on top of the other (Fig.4.A). Rendering 3D data with series of horizontal 2D planes could bring a problem of occlusion between planes, such as climate values in the middle 

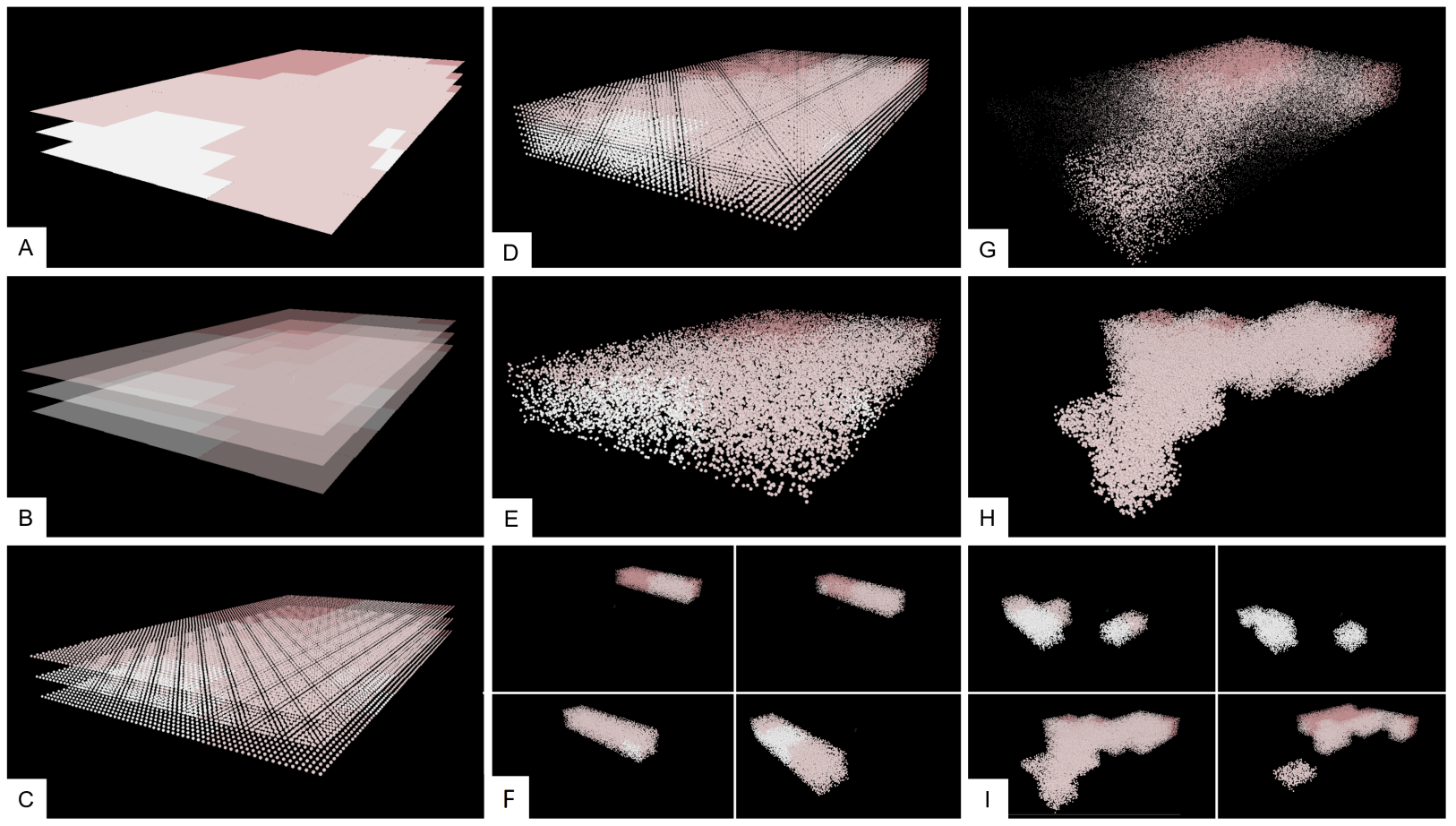

Figure 4. Representing 3D climate data by series of horizontal 2D planes or by a 3D point cloud; (A) representing 3D data by series of 2D planes; (B) exploring the use of transparency when visualizing data through series of 2D planes; (C) representing 3D data by series of 2D point clouds; (D) representing 3D data by a 3D cloud of regularly spaced points; (E) representing 3D data by a 3D cloud of randomly spaced points; (F) animation of a moving slice of a 3D point cloud along a horizontal axis; $(\mathrm{G})$ varying points size and density in the 3D cloud according to climate data value; $(\mathrm{H})$ filtering points of the $3 \mathrm{D}$ cloud according to climate data value; (I) using animation to display data value intervals one after the other.

of our 3D dataset being hidden by climate values placed above or below. We explore then the use of transparency on the $2 \mathrm{D}$ horizontal planes in order to show the different values of the data (Fig.4.B).

The 3D point cloud representation consists in representing the $3 \mathrm{D}$ climate data by a point cloud filling the volume corresponding to the $3 \mathrm{D}$ data (Fig.4.B). The objective is to avoid 3D objects (buildings or represented climate data) from being hidden by the climate data representation, by letting these objects be visible through the empty space between the particles of the point cloud. As for the representation with series of $2 \mathrm{D}$ horizontal planes, we represent the data values by a color scale, which may be tuned by the user. We let the possibility to the user to dynamically calibrate the size and the density of the points in order to improve its visual analysis according to the visualization scale. Different ways are explored to construct the representation of the $3 \mathrm{D}$ dataset:

1. by a series of $2 \mathrm{D}$ horizontal planes by using a series of horizontal 2D clouds, located at the same altitude than the previous 2D horizontal planes (Fig.4.C). Each height level is represented by regularly spaced points, the number of points being the same for each level.

2. by using a 3D cloud of regularly spaced points. The point cloud fills the entire space of the corresponding 3D dataset, allowing to visualize the volume corresponding to the simulated data (Fig.4.D).

3. by a $3 \mathrm{D}$ cloud of randomly placed points, filling the volume corresponding to the 3D dataset (Fig.4.E). This representation avoids the visualization of artificial structures remind- ing aliasing effects (stripes of aligned points and blank spaces in Fig.4.D), due to the regular positions of the points in space, which could be misinterpreted by the user.

In case of very dense point clouds, we propose to use filtering and animation functions to restrain the visualization of climate data to a slice of the point cloud, orthogonal to the $x, y$ or $z$ axis, and to make this slice move along the corresponding orthogonal axis (Fig.4.F).

The value-related 3D point cloud representation consists in representing the $3 \mathrm{D}$ climate data though a point cloud, where we modify points representation or rendering according to climate data values. Through this representation, we experiment different ways to highlight extreme values and spatial gradients in the data distribution:

- representing data values simultaneously with color, and with point size and density, by representing the high (or low) values with a higher (or lower) density of points, and/or with a bigger (or smaller) point size (Fig.4.G);

- filtering the displayed data according to their value (Fig.4.H);

- using animation to perform an animated filter of the data according to their value, displaying different value intervals one after the other, in order to identify potential spatial directions in the evolution of data (Fig.4.I).

\section{APPLICATION ON THE VISUALIZATION OF 3D AIR TEMPERATURE DATA.}

In this section, we present the application of our propositions to the visualization of $3 \mathrm{D}$ air temperature data obtained from 
Meteo-France. We co-visualize the climate data with a 3D urban model of Paris obtained from the IGN topographic database $^{4}$. We first describe the structure of the 3D climate data, and then the co-visualization of simulated temperature data in a 3D urban model.

\subsection{Structure of the raw climate data.}

The raw climate data are obtained with the Meso-NH (Mesoscale Non-Hydrostatic Model) and TEB (Town Energy Balance) simulation models, TEB being part of the SURFEX (Surface Externalisée) simulation model (Renard, Alonso, 2017, Pinson, Masson, 2016, Masson, 2000, Lac et al., 2018). The Meso$\mathrm{NH}$ model provides a simulation of climate data in the atmosphere for altitudes between 0 and 15451.8 meters above the ground. The SURFEX model has been developed to simulate climate data near the surface level. SURFEX simulations use specific models according to the different possible spatial environments, as the ISBA (Interaction Sol-Biosphère-Atmosphère) model for the rural areas, and the TEB for the urban areas (Renard, Alonso, 2017, Pinson, Masson, 2016). The TEB model provides simulated climate data in urban environments for altitudes between 0 and 46 meters above the ground, which overlap the first level of the Meso-NH simulated data

We focus our propositions on the visualization of simulated temperature data corresponding to the TEB simulation (six levels of data) and the two first levels of the Meso- $\mathrm{NH}$ simulation (0 to 132 meters above the ground), the first Meso-NH level (0 to 60 meters above the ground) overlapping the TEB levels. The study area is centered on the city of Paris.

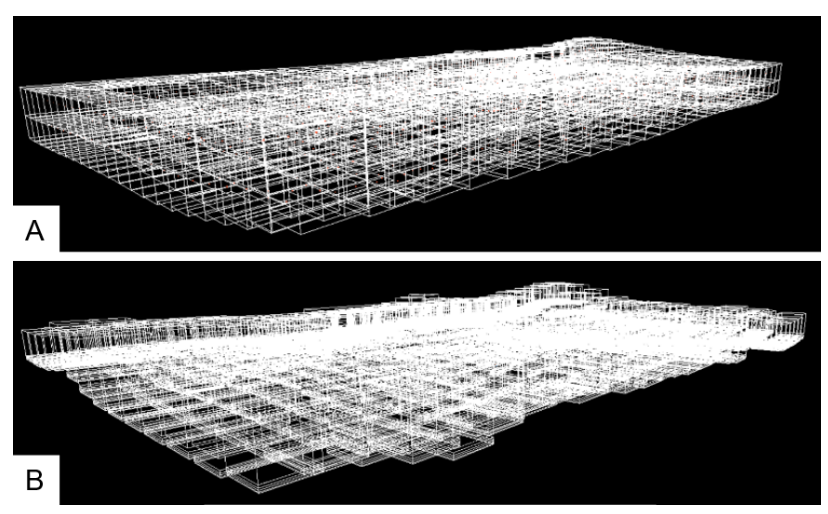

Figure 5. Visualization of the simulated climate data 3D grids; each height level is represented by a grid of 3D cells; (A) visualization of two height levels of Meso-NH data; (B) visualization of six height levels of TEB data.

The data are stored in $\mathrm{NetCDF}^{5}$ files containing simulated climate data such as temperatures, pressures, wind speed and direction, etc. and input data of the simulation process, such as vegetation ratio, the mean albedo of the buildings surface, etc. Temperature data are stored in NetCDF files as a 3D array. Each element of this array corresponds to a point located in space with geographic coordinates and a height above the ground (these altitudes are also stored in a 2D array of the NetCDF file). Each point is considered at the center of a 3D 6-faces polyedric cell. The entire array corresponds to several regular 2D grids representing the different height levels above the ground, that we have

\footnotetext{
4 geoservices.ign.fr/documentation/diffusion/documentation-offre.html 5 https://www.unidata.ucar.edu/software/netcdf/Network Common Data Form
}

represented in a 3D grid for better data representation, on which we can experiment various types of graphic representations and renderings. The Meso-NH model output can be represented as a $3 \mathrm{D}$ grid with a regular horizontal resolution of 600 meters near the ground (Fig.5.A). Along the vertical axis, the thickness of the cells is growing bigger with the height above the ground, from 60 to 700 meters height. The TEB model output can be represented as a 3D grid, which overlaps the first level of the Meso-NH 3D grid (Fig.5.B). On a horizontal plane, each TEB cell overlaps a Meso-NH cell and shares the same horizontal resolution of 600 meters. Along the vertical axis, the thickness of the cells is growing bigger with the height above the ground, from 1 to 34 meters height. In each grid, the evolution of ground altitude causes offsets of altitude between neighboring cells.

\subsection{Co-Visualization of Meso-NH air temperature data in 3D urban model.}

We present several co-visualizations of air temperature data with an urban 3D model. The urban 3D model is graphically represented in an abstract style, using the ground and roof altitudes of each building, and will not be modified. The urban model is then represented by polyedric structures, displayed with a single diffuse material (constant albedo/color). The buildings are represented with the same uniform color, and the 3D scene is lit up by two punctual lights, located at the two borders of the scene. We apply our visualization propositions at different city scales, first at a street scale, then at a city scale.

We propose to represent data values by a rainbow color scale, and different color scales (Fig.6) created with the HCL color wizard tool ${ }^{6}$.

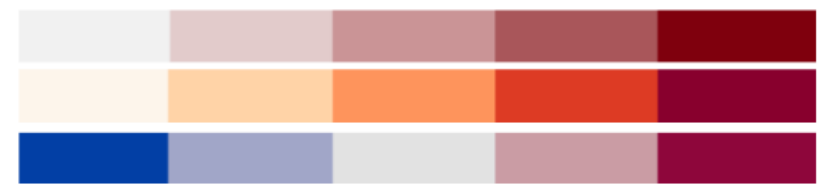

Figure 6. Examples of the used HCL color scales.

2D planes series representation. To apply our first proposition, we represent each 2D grid forming the 3D temperature grid by a $2 \mathrm{D}$ horizontal plane: this makes a stack of horizontal 2D planes, each of them corresponding to a height level above the ground. For each height level, the $2 \mathrm{D}$ grid is placed at the vertical center of the corresponding slice of the $3 \mathrm{D}$ data. The space between the 2D grids is then not regular along the vertical axis. In order to make the series of 2D grids coincide with the urban model, we represent the $2 \mathrm{D}$ grid at the corresponding altitude for each horizontal cell, using the mean altitude of the ground stored for each cell in the NetCDF file. Each 2D grid is then formed by a series of $2 \mathrm{D}$ cells, which do not share the same altitude.

The vertical thickness of 3D cells evolving from 1 to 60 meters near the ground surface, we can see an evolution of temperature along the vertical axis at a street scale. However, the representation by series of $2 \mathrm{D}$ grids makes difficult to see all the temperature values of each level, because some 2D planes are hidden by the others (Fig.7.A). The use of transparency allows to distinguish the different $2 \mathrm{D}$ planes, but brings problems for the interpretation of the color representing the temperature value, the color of one $2 \mathrm{D}$ plane being affected by the color of the $3 \mathrm{D}$

\footnotetext{
6 http://hclwizard.org:64230/hclwizard/
} 
objects behind it (Fig.7.B). Another option could be the use of animation to move one 2D horizontal plane along the vertical axis to show temperature values at different height levels, one level after the others (Fig.7.B). But it would impact the readability of the visualization.
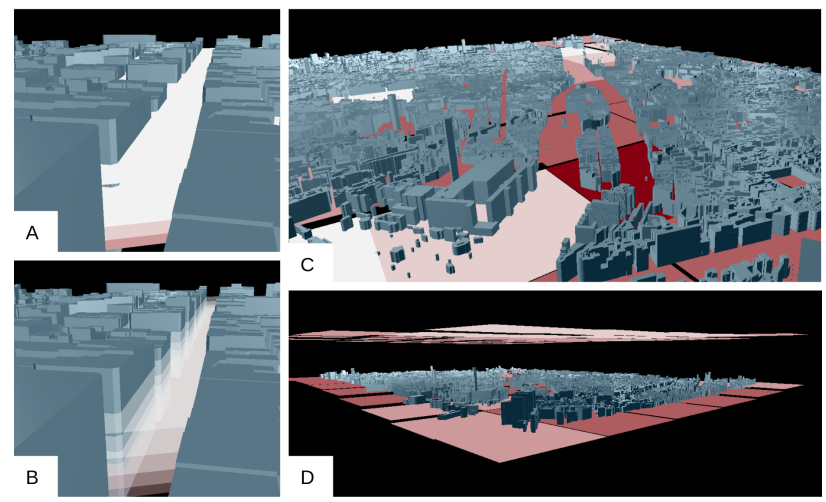

Figure 7. Visualization of air temperature data using series of 2D planes; (A) visualization at a street scale; (B) visualization at a street scale using transparency; (C) visualization of one height level of data at a city scale; (D) visualization of two height levels of data at a city scale.

The co-visualization of one $2 \mathrm{D}$ grid with the 3D city model at street and city scales shows yet the possibility to efficiently represent one 2D temperature data with the urban topography without using the building color (Fig.7.C), which could be a source of misinterpretation. This representation could be used, like in the Fig 1.A, to visualize the air temperature near the surface in order to analyze the surface UHIs, but could be also used to analyze the temperature $2 \mathrm{D}$ distribution at any specific height level calibrated by the user (Fig.7.D).

3D point cloud representation. To apply our second proposition, we represent each 3D cell of the 3D data grid by a point cloud (Fig.8.A). As for the previous 2D planes, the 3D points are represented at the corresponding altitude for each horizontal cell, using the mean altitude of the ground stored for each cell in the NetCDF file. According to the different ways of rendering the point cloud, the representation can take the shape of:

- a series of 2D horizontal clouds of regularly spaced points, located at the vertical center of the corresponding 3D cell (Fig.8.A);

- a series of 3D clouds of regularly spaced points, filling the entire volume of the corresponding 3D cell (Fig.8.B);

- a series of 3D clouds of randomly spaced points, filling the entire volume of the corresponding 3D cell (Fig.8.C).

The representation by series of 2D horizontal point clouds appears to be an alternative to the series of $2 \mathrm{D}$ planes allowing to keep the different temperature levels visible. It could be a good way to represent the evolution of the temperature values along the vertical axis without risking a cognitive overload due to a too important number of 3D graphic objects, and without misleading the user on the resolution of the displayed data. However, the representations by $3 \mathrm{D}$ point clouds allow to better distinguish the entire volume corresponding to each $3 \mathrm{D}$ cell. Among these representations, the use of regularly spaced points has the disadvantage to make visible the artificial stripes
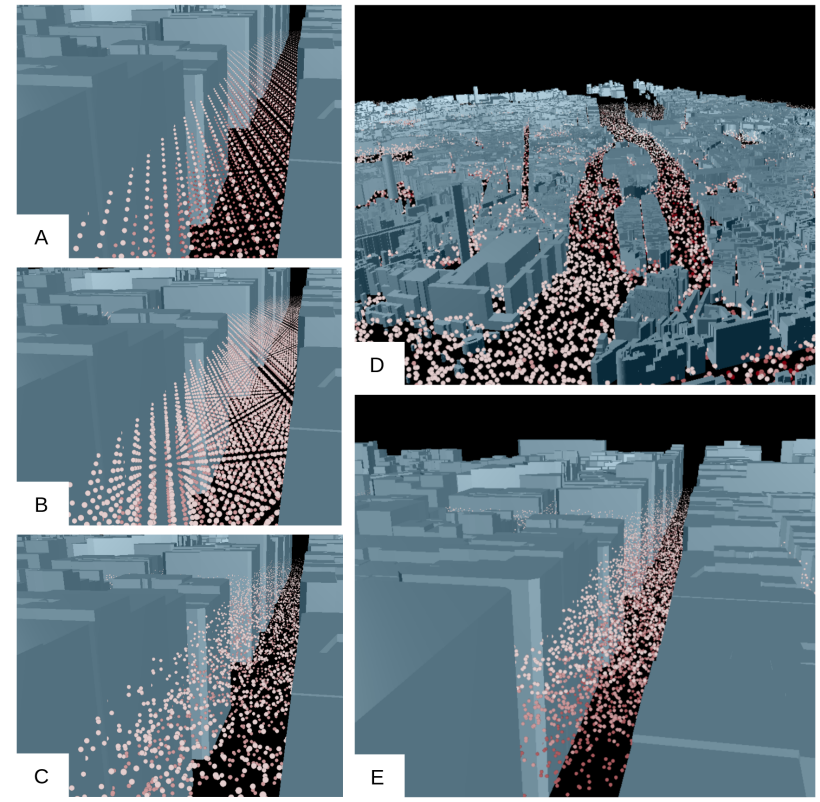

Figure 8. Visualization of air temperature data using a 3D point cloud; (A) visualization through series of 2D point clouds; (B) visualization through a 3D cloud of regularly spaced points; (C) visualization through a 3D cloud of randomly spaced points; (D) visualization at a city scale; (E) visualization at a street scale.

of aligned points reminding aliasing effects, which could mislead the user by showing him structures that do not exist.

Through these representations, we explore various settings for both points density and size. The points being difficult to distinguish when they are located far from the user points of view, we use a low density of large points in our visualizations at a global scale such as the city (Fig.8.D). At a micro-scale such as the street, we use a high density of small size points in order to let the empty spaces between points big enough to let objects being visible through it (Fig.8.E). Yet, we let the possibility to the user to dynamically calibrate the size and the density of the points in order to improve its visual analysis according to the visualization scale. Due to the better resolution of our data along the vertical axis, our representations by animated slices of points were mainly done according to the vertical axis. This representation allows to focus on the different height levels of the data one after the other, and allows seeing the location of the highest temperature near the ground surface. Yet, this representation seems to suffer the same consequences regarding readability than the other visualizations using animation.

Value-related 3D point cloud representation. The different ways we experiment to highlight the extreme values and the spatial gradients in our dataset show interesting results:

- Varying the size and the density of the points according to the temperature value allows to see the global trends in the temperature distribution. A representation of the $3 \mathrm{D}$ cells corresponding to the highest temperature values with bigger points and a higher density of points improves notably the identification of the highest temperature location, mainly near the ground surface (Fig.9.A).

- Filtering the data according to the temperature value allows a finer identification of the high values, showing notably the location of the highest value near the ground sur- 
face, but also the presence and the location of columns of hot air above the buildings (Fig.9.B).

- Using the animation to display different value intervals one after the other doesn't appear to be the better way to highlight extreme values in the dataset. However, an animated filtering according to the temperature, displaying the location of the different values (from the lowest to the highest) one after the other, allows the identification of the direction of a temperature gradient: the Fig.9.C shows that the temperature above the buildings decreases when we move from the west (bottom of the image) to the east (top of the image) (Fig.9.C).
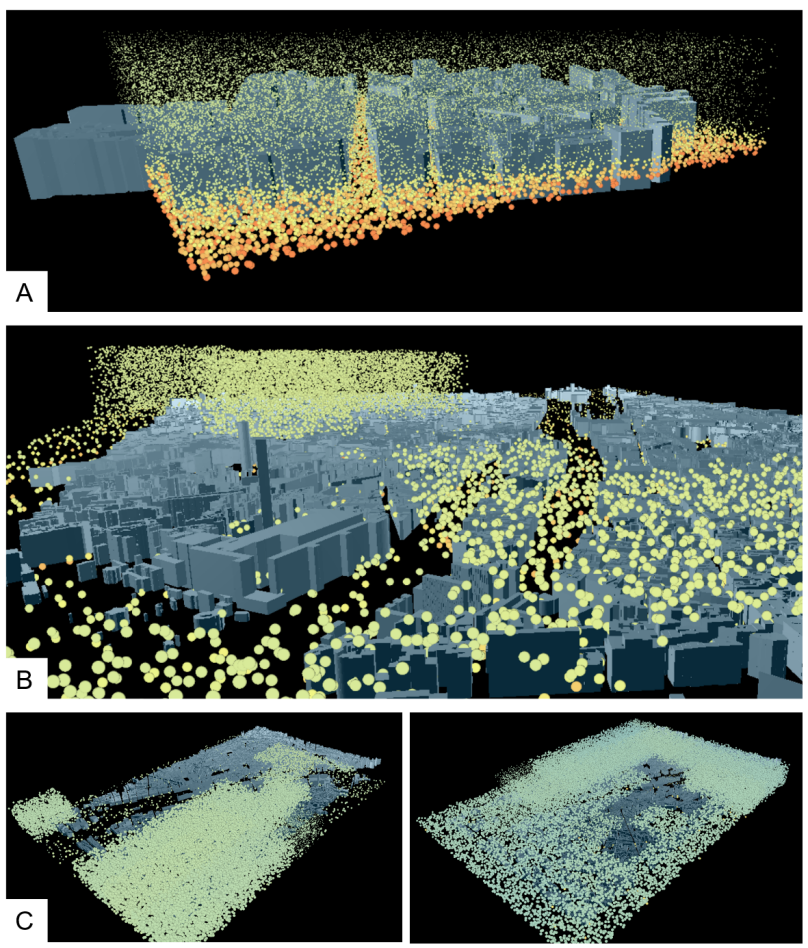

Figure 9. Exploration of different ways to highlight extreme values and spatial gradients in the temperature 3D distribution; (A) using high points size and density to represent the high values of temperature; (B) using filtering to hide the lowest values of temperature; (C) using animation to display the different temperature value intervals one after the others.

\section{DISCUSSION AND CONCLUSION.}

We experiment different ways to visualize $3 \mathrm{D}$ climate data, in order to provide a visual analysis of their distribution according to the three dimensions of space and their relationships with urban morphology, notably the structure and the shapes of the city buildings. Inspired by related works about the representation of 3D data in 3D visualization environments, we propose three main ways to visualize 3D climate data:

- through a series of 2D horizontal planes corresponding each to one height level. Data values are represented for each height level by the color of the corresponding plane.

- through a 3D point cloud filling the volume covered by the 3D data. Data values are represented by the color of the points.
- through a 3D point cloud where filtering and animation functions, or 3D semiologic rules are used to display the climate data according to their values.

For these three propositions, we represent data values with an HCL color scale using a luminance gradient. Through an application of these propositions to the co-visualization of Meso$\mathrm{NH}$ air temperature data with a 3D urban model, we explore different parameters in order to improve the efficiency of the visualizations to analyze the air temperature distribution according to the three dimensions of space. These parameters are related to the use of transparency, 3D semiologic rules, filtering and animation functions. Our first results show that our representation through 2D planes could be better used for the representation of climate data at a specific height level, while the representation by a 3D point cloud should be favored for the analysis of the entire climate data 3D distribution. 3D semiologic rules, or animated filtering of data according to their value can be used to highlight the location of extreme value or spatial gradients in the distribution of data. User tests should be done to conclude these first results. Yet, our current visualizations could be a first subject of exchange with meteorological and climate scientists. These co-visualizations are proposed to meteorological and climate scientists, in the context of the $\mathrm{URCLIM}^{7}$ project. The possibility, let to the user, to calibrate the different design parameters, allow them to define the most suitable settings for climate data analysis, according to different scales and use cases. These exchanges could lead to other co-visualizations of these simulated 3D climate data with input data, in order to help improve data simulation model.

\section{ACKNOWLEDGEMENTS.}

This research work is granted by the URCLIM project as part of ERA4CS, an ERA-NET initiated by JPI Climate with cofunding of the European Union (Grant n690462). The climate data has been provided by Valéry Masson, leader of the project.

\section{REFERENCES}

Aigner, W., Miksch, S., Schumann, H., Tominski, C., 2011. Visualization of time-oriented data. Springer Science \& Business Media.

Akbari, H., Kolokotsa, D., 2016. Three decades of urban heat islands and mitigation technologies research. Energy and Buildings, 133, 834-842.

Akbari, H., Pomerantz, M., Taha, H., 2001. Cool surfaces and shade trees to reduce energy use and improve air quality in urban areas. Solar energy, 70(3), 295-310.

Arnaud, A., Davoine, P.-A., 2009. Approche cartographique et géovisualisation pour la représentation de l'incertitude. SAGEO 2009, Nov, 2009. Paris, France, 12-32.

Borland, D., Taylor, R., 2007. Rainbow color map (still) considered harmful. IEEE computer graphics and applications, 27(2), 14-17.

Bouyer, J., 2009. Modélisation et simulation des microclimats urbains : étude de l'impact de l'aménagement urbain sur les consommations énergétiques des bâtiments. $\mathrm{PhD}$ thesis.

7 http://www.urclim.eu/ 
Bouyer, J., Musy, M., Huang, Y., Athamena, K., 2011. Mitigating Urban Heat Island Effect by Urban Design: Forms and Materials. Cities and Climate Change: Responding to an Urgent Agenda, 164-181.

Brewer, C. A., 1997. Spectral schemes: Controversial color use on maps. Cartography and Geographic Information Systems, 24(4), 203-220.

Dasgupta, A., Poco, J., Rogowitz, B., Han, K., Bertini, E., Silva, C. T., 2018. The effect of color scales on climate scientists' objective and subjective performance in spatial data analysis tasks. IEEE transactions on visualization and computer graphics, PP, 1-1.

Equere, V., Mirzaei, P. A., Riffat, S., 2020. Definition of a new morphological parameter to improve prediction of urban heat island. Sustainable Cities and Society, 56, 102-021.

Johannsen, I. M., Fabrikant, S. I., Evers, M., 2018. How do texture and color communicate uncertainty in climate change map displays? 10th International Conference on Geographic Information Science (GIScience 2018), Aug, 2018. Melbourne, Australia.

Kaddouri, L., Blaise, J.-Y., Davoine, P.-A., Mathian, H., Saint-Marc, C., 2014. État des lieux des représentations dynamiques des temporalités des territoires. Research report, UMR 7300 ESPACE ; UMR 3495 MAP - GAMSAU ; Laboratoire d'Informatique de Grenoble.

Kurppa, M., Karttunen, S., Hellsten, A., Järvi, L., 2020. Including aerosol dynamic processes in les: evaluation and application. 100th American Meteorological Society Annual Meeting, Jan, 2020. Boston, USA.

Lac, C., Chaboureau, J.-P., Masson, V., Pinty, J.-P., Tulet, P., Escobar, J., Leriche, M., Barthe, C., Aouizerats, B., Augros, C. et al., 2018. Overview of the Meso-NH model version 5.4 and its applications. Geoscientific Model Development, 11(5), 1929-1969.

Li, W., Wang, S., 2017. PolarGlobe: A web-wide virtual globe system for visualizing multidimensional, time-varying, big climate data. International Journal of Geographical Information Science, 31(8), 1562-1582.

Lobo, M.-J., Hurter, C., 2019. Data multiplexing through animated texture orientation and color. VISIGRAPP 2019 - Proceedings of the 14th International Joint Conference on Computer Vision, Imaging and Computer Graphics Theory and Applications, 3, Feb, 2019. Prague, Czech Republic., 289-296.

Masson, V., 2000. A physically-based scheme for the urban energy budget in atmospheric models. Boundary-layer meteorology, 94(3), 357-397.

Masson, V., Marchadier, C., Adolphe, L., Aguejdad, R., Avner, P., Bonhomme, M., Bretagne, G., Briottet, X., Bueno, B., De Munck, C. et al., 2014. Adapting cities to climate change: A systemic modelling approach. Urban Climate, 10, 407-429.

Oke, T. R., 1976. The distinction between canopy and boundary-layer urban heat islands. Atmosphere, 14(4), 268277.

Oke, T. R., 2002. Boundary layer climates. Routledge.
Parsaee, M., Joybari, M. M., Mirzaei, P. A., Haghighat, F., 2019. Urban heat island, urban climate maps and urban development policies and action plans. Environmental technology \& innovation, 100-341.

Pinson, L., Masson, V., 2016. Heat stress in urban area: data fusion of observations, modeling and geospatial information. First International Conference on Urban Physics (FICUP 2016), Sept, 2016. Quito, Ecuador.

Plumejeaud-Perreau, C., Poitevin, C., Pignon-Mussaud, C., Long, N., 2015. Building local climate zones by using socioeconomic and topographic vectorial databases. 9th International Conference on Urban Climate jointly with 12th Symposium on the Urban Environment, Jul, 2015. Toulouse, France.

Ramyar, R., Zarghami, E., Bryant, M., 2019. Spatio-temporal planning of urban neighborhoods in the context of global climate change: Lessons for urban form design in Tehran, Iran. Sustainable Cities and Society, 51, 101-554.

Rautenhaus, M., Böttinger, M., Siemen, S., Hoffman, R., Kirby, R. M., Mirzargar, M., Röber, N., Westermann, R., 2017. Visualization in meteorology - a survey of techniques and tools for data analysis tasks. IEEE Transactions on Visualization and Computer Graphics, 24(12), 3268-3296.

Renard, F., Alonso, L., 2017. La combinaison de l'image satellitaire avec les données citoyennes pour la mesure de l'îlot de chaleur urbain. Premiers résultats sur la métropole de Lyon. Ingenierie des Systemes d'Information, 22(5), 105.

Rodler, A., Leduc, T., 2019. Local climate zone approach on local and micro scales: Dividing the urban open space. Urban Climate, 28, 100-457.

Santamouris, M., 2001. Heat-island effect. Energy and climate in the urban built environment, 402.

Schneider, B., Nocke, T., 2018. The feeling of red and blue-a constructive critique of color mapping in visual climate change communication. Handbook of Climate Change Communication: Vol. 2, Springer, 289-303.

Spur, M., Tourre, V., 2018. Evaluation of spatial data layer compositing techniques in interactive vr environments. VGI Geovisual Analytics Workshop, 4th International Symposium on Big Data Visual and Immersive Analytics, Oct, 2018. Konstanz, Germany.

Stauffer, R., Mayr, G. J., Dabernig, M., Zeileis, A., 2015. Somewhere over the rainbow: How to make effective use of colors in meteorological visualizations. Bulletin of the American Meteorological Society, 96(2), 203-216.

Stewart, I. D., Oke, T. R., 2012. Local climate zones for urban temperature studies. Bulletin of the American Meteorological Society, 93(12), 1879-1900.

Stewart, I., Oke, T., 2009. Classifying urban climate field sites by "local climate zones": The case of nagano, japan. 7th International Conference on Urban Climate (ICUC-7), (29), JunJul, 2009. Yokohama, Japan.

Ware, C., Bolan, D., Miller, R., Rogers, D. H., Ahrens, J. P., 2016. Animated versus static views of steady flow patterns. ACM Symposium on Applied Perception, July, 2016. Anaheim, USA., 77-84. 